J. Astron. Space Sci. 20(2), 95-100 (2003)

\title{
RELATIONSHIP BETWEEN GEOMAGNETIC STORMS AND RELATIVISTIC ELECTRON EVENTS
}

\author{
Hee-Jeong $\mathrm{Kim}^{\dagger}$, and Dae-Young Lee \\ Department of Astronomy and Space Science, \\ College of Natural Sciences and Institute for Basic Science Research, \\ Chungbuk National University, Chungju, Korea \\ email: heekim@chungbuk.ac.kr, dylee@chungbuk.ac.kr
}

(Received March 23, 2003; Accepted May 23, 2003)

\begin{abstract}
This paper is for the investigation of the relationship between the geomagnetic disturbances and the relativistic electron events occurring at geosynchronous orbit. We have analyzed the electron fluxes of $E>2 \mathrm{MeV}$ measured by GOES 10 satellite and the hourly Dst index for the period of April, 1999 to December, 2002. With the rigorous definition of the relativistic event, total 34 events were identified during the time period. Our statistical study showed that more than $50 \%$ of the total events occurred associated with weak (or sometimes virtually no) magnetic storms. And only 20\% of the events took place accompanied by a strong magnetic storm of Dst $t_{\min }<-100$ $n T$. This result suggests that large geomagnetic storms may not be crucial for the occurrence of a relativistic event at geosynchronous orbit. We also found that there is no clear correlation between the maximum electron flux of an event and the associated minimum of Dst. Therefore any study on the physical mechanism (s) accounting for the relativistic events should take it into account that strong magnetic storms may not be necessarily required for the occurrence of a relativistic electron event at geosynchronous orbit.
\end{abstract}

Keywords : magnetic storms, relativistic electron events, outer-belt $\mathrm{MeV}$ electrons

\section{INTRODUCTION}

The intensity of outer belt $\mathrm{MeV}$ electrons often highly fluctuates during geomagnetically disturbed times. It has been reported that a prolonged high intensity of the relativistic electrons, socalled relativistic electron event, can damage electronic systems in operating satellites (Baker et al. 1994, Gussenhoven et al. 1991). Many studies have been devoted to understanding the physical mechanisms that account for the large variability of the outer radiation belt relativistic electrons, but it still remains as an unsolved important topic (see the references in Kim et al. (2000)).

It has been a general belief that relativistic electron events occur after a strong geomagnetic storm. Accordingly, most of the proposed scenarios for the relativistic electron events have usually

\footnotetext{
${ }^{\dagger}$ corresponding author
} 
assumed large geomagnetic perturbations as a necessary condition. However, careful examinations of the variation of relativistic fluxes can reveal that it is not uncommon to observe a relativistic electron event without accompanying a large magnetic storm. In the present study, we investigate the statistical relationship between the occurrence of a relativistic electron event at geosynchronous orbit and the magnetic storms. The development of a magnetic storm is usually characterized by the Dst ("storm-time disturbance") index (Su \& Konradi 1975). Dst is constructed from a set of lowaltitude magnetograms. It gives an indication of the average reduction of the horizontal component of the geomagnetic field due to the ring current. When the ring current is enhanced Dst is negative and a larger negative Dst means a more intense storm.

Previously Reeves (1998) have examined the relationship between relativistic electron enhancements at geosynchronous orbit and magnetic storms measured by the Dst index using LANL geostationary satellite data. By correlating the maximum electron flux in each event with the minimum Dst value they found the correlation between the strength of a magnetic storm and the strength of the relativistic electron event is relatively low and the relativistic electron events were only observed during magnetic storms.

In this paper we conduct a similar study to Reeves (1998), adopting a more rigorous statistical approach and using the GOES 10 satellite data. The analyzed observational data exhibit some relativistic electron event occurred without accompanying geomagnetic storms. It indicates that a relativistic electron event does not necessarily occur associated with a geomagnetic storm, which is inconsistent with Reeves (1998). We also find no cbvious correlation between the maximum electron flux of an event and the size of geomagnetic storm similar to Reeves (1998). Therefore it is conceivable that the role of a geomagnetic disturbance for the relativistic electron enhancements at geosynchronous could be less essential than commonly presumed.

Following sections present the results of the data analysis and discuss their significance in the study of relativistic electron events.

\section{DATA ANALYSIS AND RESULTS}

In order to find the relativistic electron events occurring at geosynchronous orbit, we analyze the 5-minute average electron fluxes of $E>2 \mathrm{MeV}$ measured by the GOES 10 satellite. The electron fluxes are taken from the CDA (Coordinated Data Analysis) web data service located by the URL http://cdaweb.gsfc.nasa.gov/. When a daily average flux is continuously larger than $2 \times 10^{3} \mathrm{~cm}^{-2} \mathrm{~s}^{-1} \mathrm{sr}^{-1}$ for at least 3 days, we define it as the relativistic electron event. This base level is determined referring to the electron flux measurements that were associated with two satellite failures occurred in 1998 [ see Figure 9 in Baker (2000)].

The daily average flux is calculated by taking an average over the fluxes measured at local noon and midnight of each day. If the time interval between the two events is less than 2 days, they are counted as one event. During the examined time period, total 34 relativistic events are identified; 10 events for 1999, 11 events for 2000, 4 events for 2001, and 9 events for 2002.

Next is to obtain the strength of the geomagnetic disturbance that precedes a relativistic event. Using the hourly Dst index, we find the minimum Dst values corresponding to each event within 3 days prior to the relativistic electron event, as the electron flux usually reaches the based level in a few days after the storm main phase.

Figure 1 shows two examples of the relativistic events in year 2000. Event 1 occurred on Feb. 25 with no preceding strong magnetic storm (the associated Dst minimum is merely $-27 \mathrm{nT}$ ), while Event 2 on Apr. 10 is associated with the magnetic storm of Dst $t_{\min }=-321 \mathrm{nT}$. Table 1 presents the results of the data analysis for the fifteen sample relativistic events which are arbitrarily chosen 


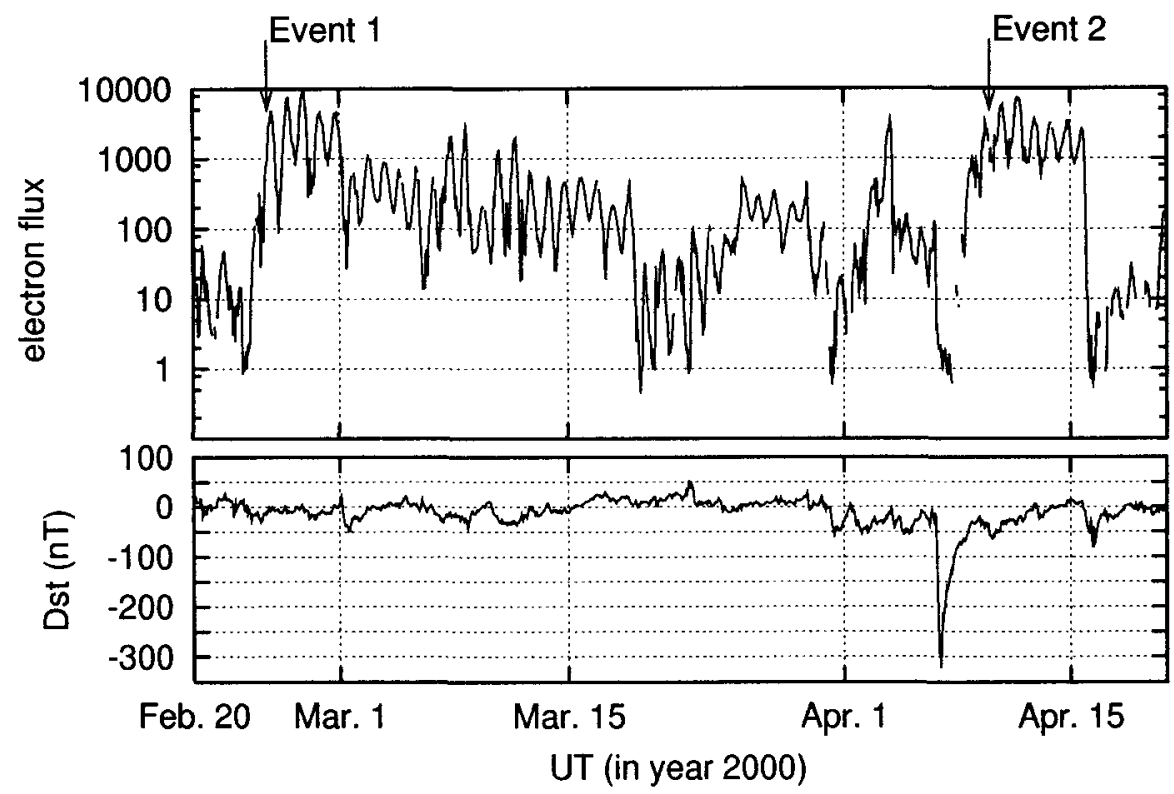

Figure 1. (a) Variation of hourly average electron fluxes for $E>2 \mathrm{MeV}$ measured by the GOES 10 satellite. Two arrow indicate the relativistic electron events occurred on Feb. 25 and Apr. 10, respectively. (b) Changes of the hourly Dst index.

among the total events. From the left to the right, each column in the table denotes the starting date of the event, the event duration, the maximum electron flux, which is obtained from the maximum flux at local noon during the event, the average electron flux of the event, and the associated Dst minimum, respectively.

The average electron flux over the total identified events is about $6785 \mathrm{~cm}^{-2} \mathrm{~s}^{-1} \mathrm{sr}^{-1}$ and the average duration of one event is approximately 6 days, spanning from minimum 3 days (e.g., Sep. 21,2000 ) to maximum 11 days (e.g., Aug. 27, 1999). The minimum values of Dst vary from $-7 \mathrm{nT}$ to $-321 \mathrm{nT}$, exhibiting a large variation depending on events. The maximum electron flux for each event also varies in a broad range of $5235 \sim 58525 \mathrm{~cm}^{-2} \mathrm{~s}^{-1} \mathrm{sr}^{-1}$. Figures 1 through 3 visualize the results in Table 1.

Figure 2 shows the distribution of the minimum Dst values associated with the relativistic events. About $53 \%$ of the events took place for $\mathrm{Dst}_{\min } \geq-50 \mathrm{nT}$ which can be regarded as weak storms or sometimes virtually no magnetic storms. And only about $20 \%$ of the events occurred with Dst $_{\min }<-100 \mathrm{nT}$ which represents a large storm. These results, therefore, indicate that a majority of the relativistic events occurred during the examined time period are associated with moderately large to very weak magnetic disturbances. It is contrary to the prevailing presumption of the strong dependence of the relativistic events on large magnetic storms.

The maximum electron fluxes versus the associated Dst minima are plotted in Figure 3(a). As shown in the plot, the maximum electron fluxes scatter in a wide range of the minimum Dst values, exhibiting no apparent correlation beween them. This result is consistent to the study by Reeves (1998). Similarly, no obvious correlation is found between the average electron fluxes and minimum 


\section{$98 K I M \& L E E$}

Table 1. Characteristics of the 15 relativistic electron events that are arbitrarily sampled from the total events occurred during April, 1999 through December, 2002. Fluxes are in the unit of $\# \mathrm{~cm}^{-2} \mathrm{~s}^{-1} \mathrm{sr}^{-1}$.

\begin{tabular}{|c|c|c|c|c|}
\hline starting date & duration (days) & maximum flux & average flux & minimum Dst (nT) \\
\hline Apr. 3, 1999 & 5 & 7756 & 3066 & -46 \\
\hline Aug. 17, 1999 & 6 & 58525 & 16524 & -36 \\
\hline Sep. 13,1999 & 8 & 31032 & 6202 & -50 \\
\hline Oct. 11,1999 & 10 & 16301 & 4977 & -84 \\
\hline Nov. 9,1999 & 5 & 14093 & 6021 & -81 \\
\hline Jan. 1,2000 & 9 & 21332 & 6193 & -46 \\
\hline Feb. 7,2000 & 10 & 15296 & 4385 & -39 \\
\hline Apr. 10,2000 & 5 & 6520 & 2797 & -321 \\
\hline Sep. 21,2000 & 3 & 5338 & 2641 & -170 \\
\hline Dec. 11,2000 & 5 & 5235 & 2760 & -26 \\
\hline Apr. 14,2001 & 4 & 29937 & 14707 & -256 \\
\hline Jul. 19, 2001 & 3 & 6462 & 3199 & -23 \\
\hline Aug. 13, 2002 & 6 & 9555 & 3006 & -36 \\
\hline Oct. 4,2002 & 3 & 10450 & 4410 & -179 \\
\hline Nov. 5,2002 & 5 & 8967 & 3684 & -67 \\
\hline
\end{tabular}
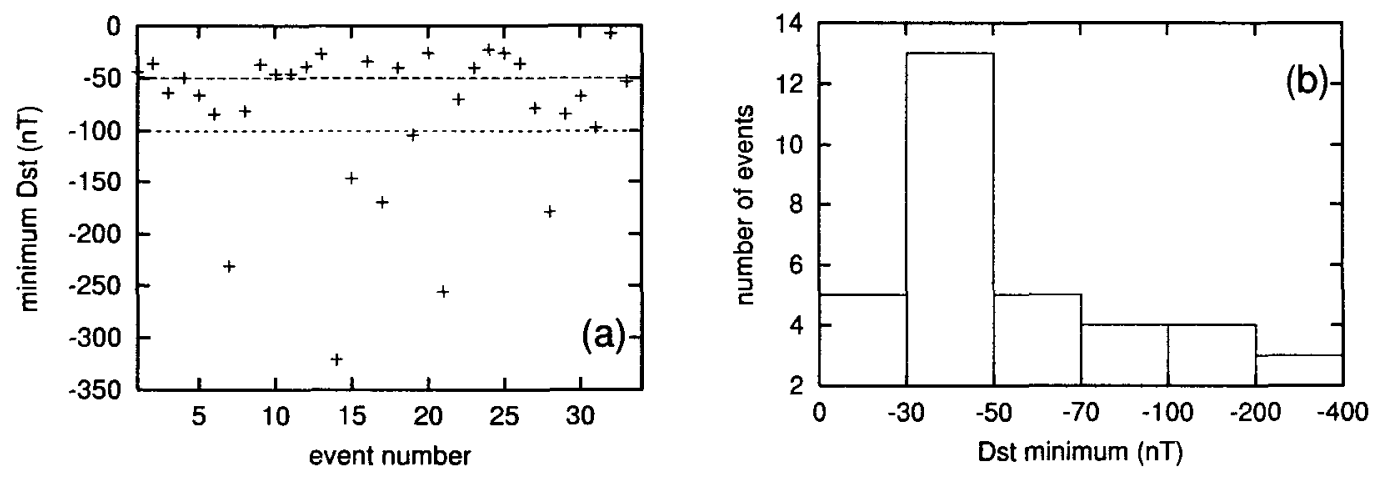

Figure 2. (a) Distribution of the minimum of Dst corresponding to each event. Two dotted lines denote the minimum Dst of $-50 \mathrm{nT}$ and $-100 \mathrm{nT}$, respectively. (b) Histogram of the number of the relativistic events versus the interval of the minimum Dst values.

Dst values, as illustrated in Figure 3(b).

It is also an interesting question how frequently large magnetic storms take place accompanying a relativistic event at geosynchronous orbit. For the estimation, we count the number of the magnetic storms with Dst $_{\min }<-100 \mathrm{nT}$ during the same time period, and then find how many of them are followed by a relativistic event. Total 33 large magnetic storms are identified, and for ten storms out of them accompany a relativistic electron event. This means that only about $30 \%$ of the large magnetic storms occurred in association with the enhancements of relativistic electron fluxes at geosynchronous orbit. It is yet to be studied why some storms have a relativistic event and others do not, if magnetic storms are relevant to the occurrence of relativistic events. 

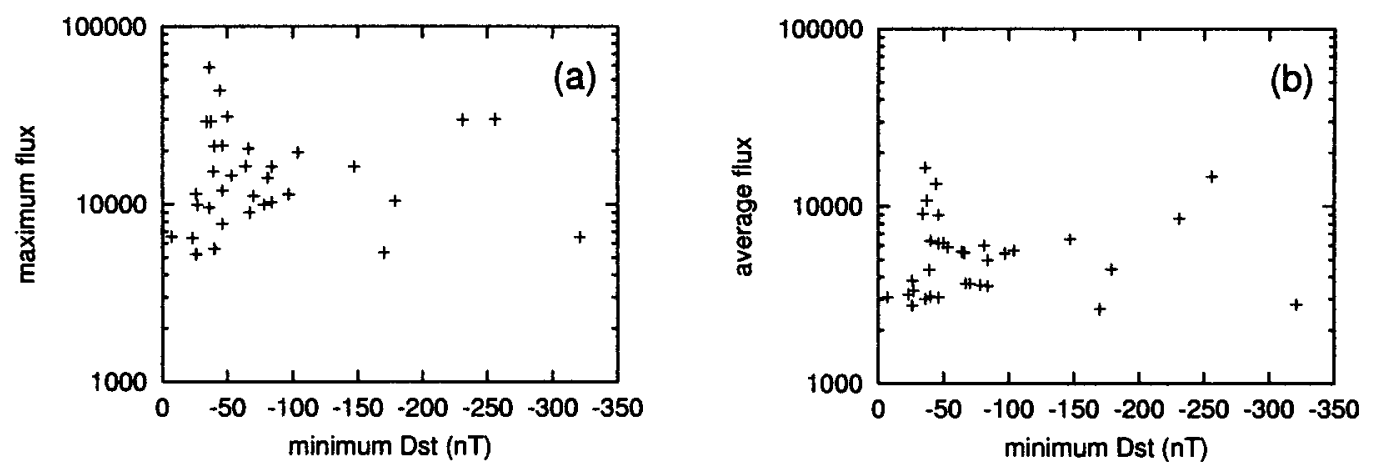

Figure 3. (a) Maximum electron flux in each event versus associated minimum of Dst. (b) Average electron flux of each event versus associated minimum of Dst.

\section{CONCLUSION AND DISCUSSION}

We have shown that the relativistic electron events at geosynchronous orbit do not always occur associated with strong geomagnetic storms. Analyzing the electron fluxes measured by the GOES 10 satellite, 34 events were identified during the period of April, 1999 through December, 2002. More than $50 \%$ of the events are found to be in association with weak (or sometimes negligible) magnetic disturbances of Dst $t_{\min } \geq-50 \mathrm{nT}$. Furthermore, the strength of a relativistic electron event does not show any obvious correlation with the size of the associated geomagnetic storm. The same analysis with the electron data for $E>0.6 \mathrm{MeV}$ measured by GOES 10 satellite yields the similar results. Although we obtain a larger number of relativistic electron events with a different base level for a different energy channel, the fractions of the events associated with certain magnetic storm sizes do not show a noticeable energy dependence. One can therefore conclude that strong geomagnetic disturbances may not be essential for the relativistic electron event at geosynchronous orbit, and also the strength of the event is irrelevant to the size of geomagnetic disturbance.

Despite that the large flux enhancements for a relativistic event requires non-adiabatic process (es), the sudden drops of electron flux by orders of magnitude during the storm main phase is understood in terms of fully-adiabatic effect (or so-called Dst effect) (Kim \& Chan 1997). When a magnetic storm develops, relativistic electrons respond to the magnetic field deflection due to the enhanced ring currents by conserving all three adiabatic invariants. Because of gradients in the particle phase space density, the electron flux in a given energy channel typically decreases during the storm main phase. It then usually increases back to pre-storm level during the recovery phase, unless strong non-adiabatic loss or source processes take place.

While the association of geomagnetic storms with relativistic electron events is puzzling, the solar wind variations are found to be closely related with the relativistic events at geosynchronous orbit. The recent study by $\mathrm{Kim}$ et al. (2003) has analyzed the electron fluxes of $E>2 \mathrm{MeV}$ measured by GOES 10 and the solar wind parameters by ACE satellite. They found that, regardless of the existence of the preceding strong magnetic storms, the relativistic electron events are accompanied by extremely quiet solar wind conditions which can be characterized as low solar wind density, weak magnetic field, and fast Alfvenic fluctuations in IMF $B_{z}$ with the small amplitude. The extremely quiet conditions mean no strong dynamic pressure and no significant southward turnings of IMF $B_{z}$, 
which yield less disturbed inner magnetosphere. In the stable and more dipole-like magnetospheric configurations, the newly generated relativistic electrons (if any) can be trapped and effectively accumulated to yield a high electron flux.

Therefore, it can be concluded from the present study and the study by Kim et al. (2003) that solar wind conditions may play more important role in the relativistic events at geosynchronous orbit compared with magnetic storms. It is also presumable that acceleration process(es) of energetic electrons may act on more frequently without leading to a relativistic event unless the specific solar wind conditions are satisfied so that the accelerated. electrons can be kept in a trapped region for a long period.

Any study on the physical mechanism (s) accounting for the relativistic electron events, therefore, should consider at least the results summarized as follows: 1) Where is no strong correlation between the relativistic events and the geomagnetic storms, and 2) extremely quiet solar wind condition is necessary for the occurrence of relativistic event at geosynchronous orbit.

ACKNOWLEDGEMENTS: This work was supported by grant No. (R01-2002-000-00100-0(2002)) from the Basic Research Program of the Korea Science \& Engineering Foundation.

\section{REFERENCES}

Baker, D. N., Kanekal, S., Blake, J. B., Klecker, B., \& Rostoker, G. 1994, Eos Trans. AGU, 75, 404 Baker, D. N. 2000, IEEE Trans. Plas. Sci., 28, 2007

Gussenhoven, M. S., Mullen, E. G., Brautigam, D. H., Holeman, E., \& Jordan, C. 1991, IEEE Trans. Plas. Sci., 38,1655

Kim, H.-J., \& Chan, A. A. 1997, JGR, 102, 22107

Kim, H.-J., Chan, A. A., Wolf, R. A., \& Birn, J. 20C0, JGR, 105, 7721

Kim, H.-J., Rostoker, G., \& Kamide, Y. 2003, in preparation

Reeves, G. D. 1998, GRL, 25, 1817

Su. S. Y.. \& Konradi. A. 1975. JGR. 80. 195 